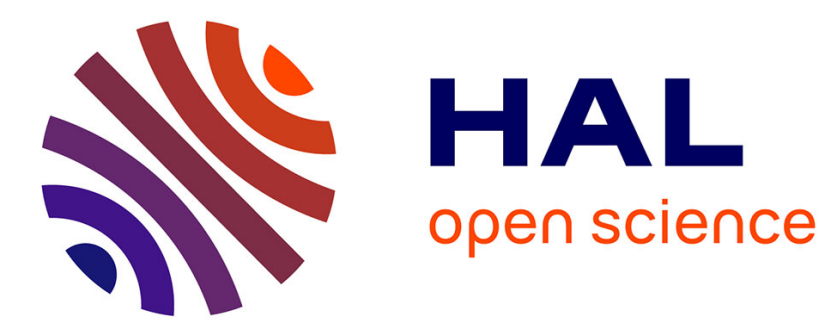

\title{
Un livre d'heures inédit de l'atelier de Simon Marmion à Valenciennes \\ Marc Gil
}

\section{To cite this version:}

Marc Gil. Un livre d'heures inédit de l'atelier de Simon Marmion à Valenciennes. Revue de l'Art, 1998, 121 (1), pp.43 - 48. 10.3406/rvart.1998.348401 . hal-01609893

\section{HAL Id: hal-01609893 \\ https://hal.univ-lille.fr/hal-01609893}

Submitted on 9 Oct 2017

HAL is a multi-disciplinary open access archive for the deposit and dissemination of scientific research documents, whether they are published or not. The documents may come from teaching and research institutions in France or abroad, or from public or private research centers.
L'archive ouverte pluridisciplinaire HAL, est destinée au dépôt et à la diffusion de documents scientifiques de niveau recherche, publiés ou non, émanant des établissements d'enseignement et de recherche français ou étrangers, des laboratoires publics ou privés. 


\section{Un livre d'heures inédit de l'atelier de Simon Marmion à}

\section{Valenciennes}

In: Revue de l'Art, 1998, n¹21. pp. 43-48.

Citer ce document / Cite this document :

Gil Marc. Un livre d'heures inédit de l'atelier de Simon Marmion à Valenciennes. In: Revue de l'Art, 1998, n¹21. pp. 43-48.

doi : 10.3406/rvart.1998.348401

http://www.persee.fr/web/revues/home/prescript/article/rvart_0035-1326_1998_num_121_1_348401 


\section{Un livre d'heures inédit de l'atelier de Simon Marmion à Valenciennes}

Le colloque sur Simon Marmion, organisé en 1990 par le J. Paul Getty Museum, a permis d'éclaircir de nombreux problèmes soulevés autour de ce peintre depuis une trentaine d'années'. Une des contributions les plus décisives fut celle de Sandra Hindman, tendant à identifier deux feuillets découpés comme provenant du Bréviaire de Charles le Téméraire?. Sa proposition, très convaincante, rend définitivement à Marmion l'ensemble des œuvres groupées autour du Retable de Saint-Bertin. Nous voudrions ajouter à la connaissance du fonctionnement de l'atelier de Simon Marmion à Valenciennes ${ }^{3}$, l'étude d'un nouveau manuscrit. Il s'agit d'un petit livre d'heures ${ }^{4}$, que la $\mathrm{Bi}$ bliothèque nationale de France a acquis sur le marché parisien, fin $1994^{5}$. Cette nouvelle acquisition permet à la Bibliothèque nationale de posséder une des rares preuves concrètes de l'existence de l'atelier du grand peintre, installé à Valenciennes depuis au moins 1458 . Ce livre apparaît également comme un jalon essentiel dans la compréhension de l'enluminure en Hainaut après la mort de Marmion. L'histoire de ce manuscrit est très courte. Conservé par un particulier, sans doute dans le département du Nord jusqu'à sa vente récente, il se trouvait aux XVIII et XIX siècles à Fei- gnies, petite ville sur la route de Valenciennes, à cinq kilomètres à l'ouest de Maubeuge 6 . Nous ne savons rien sur la période antérieure au XVIII" siècle.

Ce codex suit la tradition éditoriale en vogue en Picardie et dans les Pays-Bas du sud, en matière de livres d'heures. Après le calendrier, les Heures de la Croix sont suivies des Heures du Saint-Esprit, puis de l'Office de la Vierge ${ }^{7}$. A la manière flamande, chacune des douze miniatures est peinte à pleine page sur le verso d'un feuillet au recto blanc, mais seulement dix sont sur un feuillet isolé ajouté au cahier par un talon. Les deux autres appartiennent à un bifolio et le texte de prime se termine au recto de l'Annonce aux bergers. Suivant les habitudes françaises, le cycle de peintures qui illustre le petit office de la Vierge s'achève par le Couronnement de la Vierge et la miniature de David en prière introduit les psaumes de la pénitence ${ }^{8}$. Mais la Nativitéet l'Annonce aux bergers sont inversées, une anomalie qui ne se rencontre, à notre connaissance, que dans trois livres d'heures de Simon Marmion" et dans les Heures de Jacques de Rambures, manuscrit éponyme du Maître de Rambures, enlumineur anonyme picard ${ }^{10}$.

L'office de la Vierge suit l'usage de Rome, mais les vigiles des morts représentent celui de Sarum (Salisbury), ce qui est assez incompréhensible pour un manuscrit dont le calendrier et les litanies sont à l'usage du nord de la France et peutêtre même de Valenciennes. L'intérêt de ce calendrier réside dans ses ajouts; en effet, à côté de la partie originelle et sur les mêmes feuillets, une main du XV siècle a inscrit de nombreux saints. Le calendrier d'origine possède quelques saints propres au nord de la France et à la Flandre ${ }^{11}$. Les noms ajoutés appartiennent tout à la fois aux diocèses d'Amiens, de Thérouanne, d'Arras et de Cambrai ${ }^{12}$. Les litanies, quant à elles, contiennent deux saints de Picardie et d'Artois et plusieurs des Pays-Bas méridionaux ${ }^{13}$.

Par sa composition, ordre des rubriques, manière d'insérer les miniatures et contenu des litanies et du calendrier en français ${ }^{14}$, ce livre d'heures était donc destiné au marché des provinces francophones des Pays-Bas bourguignons. Nous ne connaissons pas de livres d'heures pour Valenciennes qui, au $\mathrm{XV}^{\circledR}$ siècle, dépendait à la fois des diocèses d'Arras et de Cambrai. Calendrier et litanies reflètent peut-être cette situation particulière.

La médiocre qualité du support, un parchemin rugueux et gris, est compensée par la qualité de l'ornementation. L'utilisation de la même 

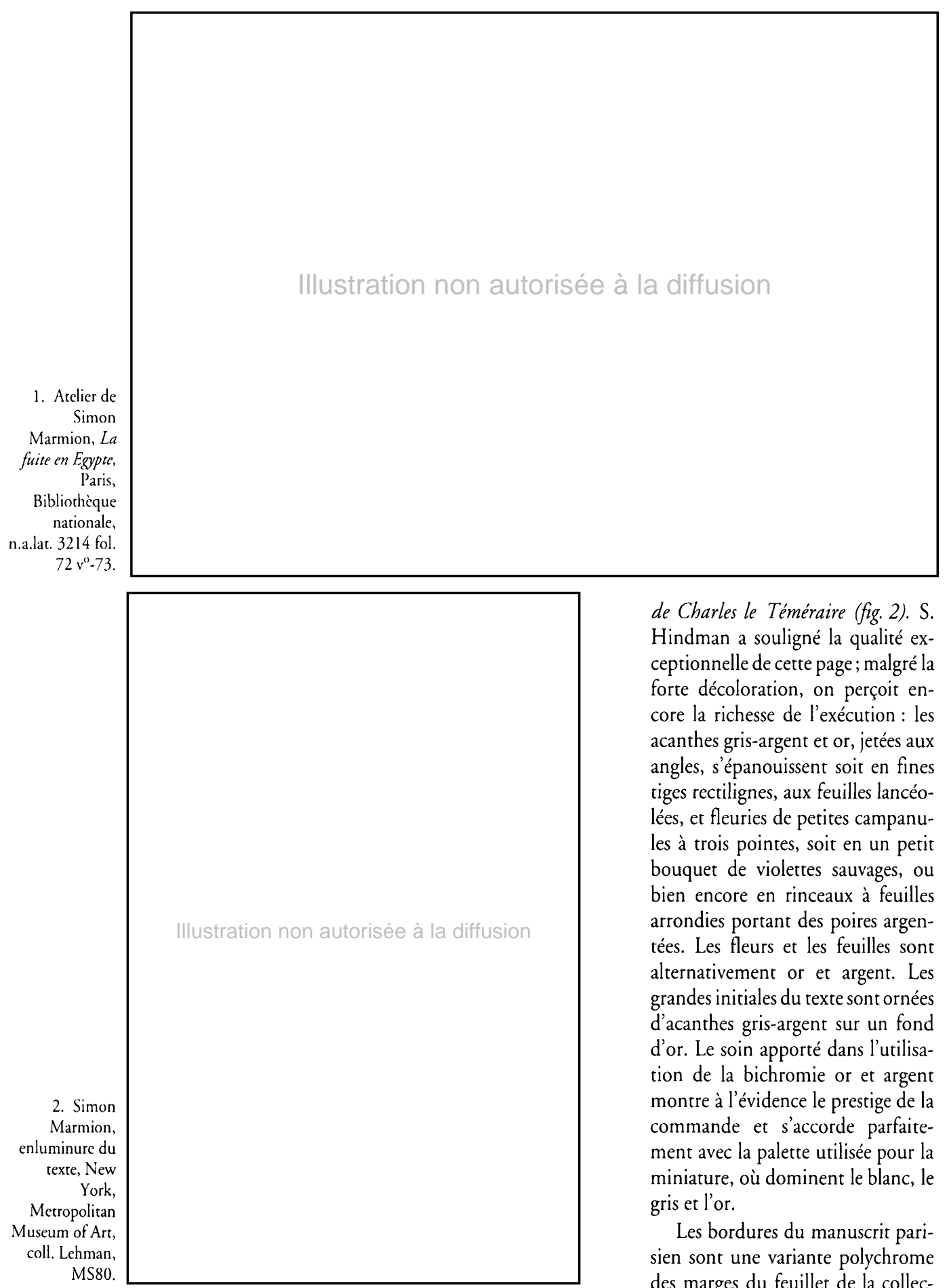

palette pour les miniatures et les bordures (ou vignettes) et le travail conjoint du miniaturiste et du vigneteur dans les encadrements comme dans les grandes initiales incitent à penser que l'ensemble de la décoration a été exécuté dans un même atelier.

Le beau décor marginal qui encadre les miniatures et leurs contrefeuillets et dans lequel prédomine encore un fond dense de fins rinceaux noirs, piquetés d'or, et le de Charles le Téméraire (fig. 2). S. Hindman a souligné la qualité exceptionnelle de cette page; malgré la forte décoloration, on perçoit encore la richesse de l'exécution: les acanthes gris-argent et or, jetées aux angles, s'épanouissent soit en fines tiges rectilignes, aux feuilles lancéolées, et fleuries de petites campanules à trois pointes, soit en un petit bouquet de violettes sauvages, ou bien encore en rinceaux à feuilles arrondies portant des poires argentées. Les fleurs et les feuilles sont alternativement or et argent. Les grandes initiales du texte sont ornées d'acanthes gris-argent sur un fond d'or. Le soin apporté dans l'utilisation de la bichromie or et argent montre à l'évidence le prestige de la commande et s'accorde parfaitement avec la palette utilisée pour la miniature, où dominent le blanc, le gris et l'or.

Les bordures du manuscrit parisien sont une variante polychrome des marges du feuillet de la collec-

jeu d'entrelacs symétriques de grosses acanthes fleuries, bleues, rouge, ocre, gris-argent et vertes (fig. 1, 4, 14), suit la tradition des manuscrits enluminés dans le nord de la France vers 1460-1465, mais aussi celle des manuscrits picards du deuxième quart $\mathrm{du} \mathrm{XV}^{\mathrm{e}}$ siècle. Cette ornementation, assez chargée, peut être rapprochée de celle qui accompagne le texte au recto du feuillet provenant du Bréviaire

3. Maitre des Ethiques d'Aristote, détail de la bordure inférieure, Rouen, Bibliochèque municipale, ms, 1., 2, fol. $17 \mathrm{v}^{0}$. tion Lehman ${ }^{15}$. On retrouve le même type de tiges aux feuilles lancéolées et aux clochettes à trois pointes, les rinceaux ornés de feuilles rondes et alourdis de poires, le jeu sur la bichromie pour le feuillage, ici vert et ocre, l'alternance des couleurs pour les campanules (fig. I) et l'utilisation du même motif dans la bordure inférieure du Couronnement de la Vierge, inversé par rapport au feuillet isolé: une acanthe s'épanouissant en une tige de violette sauvage. Enfin les deux vigneteurs utilisent la même technique de report de poncif par dessin perforé : certains feuillages sont cernés de pointillés noirs. Cette similitude de technique et de style nous laisse penser que la décoration secondaire des deux manuscrits est l'œuvre du même vigneteur.

Un second rapprochement $s^{\prime}$ impose avec le décor marginal du frontispice des Grandes Chroniques de France, chef-d'œuvre de Simon Marmion. Ce manuscrit destiné à Philippe le Bon fut terminé en 1457 ou $1458^{16}$. Comme pour les deux œuvres précédentes, la décoration secondaire de ce frontispice restait jusqu'à présent isolée dans l'œuvre de Marmion. Ici, bien que les acanthes soient plus acérées, nous retrouvons un schéma décoratif identique, avec le même type d'entrelacs aux acanthes fleuries et le même jeu sur la polychromie en parfait accord avec la palette du peintre principal. L'identité des figures d'hybrides, en particulier avec celles des folios $57 \mathrm{v}^{\circ}$, 73 et $86 \mathrm{v}^{\circ}$ du manuscrit parisien, trahit l'utilisation de modèles communs, mais renvoie également à la décoration marginale d'un autre manuscrit, contemporain des Grandes Chroniques, le Livre des Ethiques d'Aristote $^{17}$. La décoration de ce dernier fut commandée par l'échevinage de Rouen à un enlumineur anonyme picard, gravitant dans le cercle Marmion-Loyset Liédet et travaillant sans doute à Amiens ou peut-être à Hesdin dans la décennie cinquante. Le même modèle a servi à peindre l'hybride à tête d'homme barbu au folio 73 du manuscrit parisien et au folio $17 \mathrm{v}^{\circ}$ de celui de Rouen (fig. 1,3).

Comme la décoration du bréviaire ducal, entreprise à Valenciennes en 1467 peu avant la mort de 
Philippe le Bon, fut achevée pour son fils Charles en $1470^{18}$, on peut admettre que notre petit livre d'heures fut exécuté dans l'atelier valenciennois de Simon Marmion vers 1467, peut-être même un peu avant. Les rapports étroits qui unissent l'enluminure picarde de la décennie cinquante à notre manuscrit pourrait laisser supposer que ce dernier est bien antérieur au Bréviaire de Charles le Téméraire, datant alors de la même époque que les manuscrits de Rouen ou de Saint-Pétersbourg. En fait, l'utilisation de modèles communs pour les figures marginales montre simplement le lien naturel qui relie les ateliers picards ${ }^{19}$ à Simon Marmion, amiénois installé à Valenciennes, résultat de la circulation de modèles entre enlumineurs d'une même province et de leur survivance dans les ateliers de peintres émigrés. Une constatation que confirme à nos yeux l'étude des miniatures.

Un regard rapide sur les peintures pourrait laisser croire que nous sommes en présence d'une œuvre de Marmion lui-même, en particulier avec la Vierge à l'Enfant trônant entre deux anges musiciens, peinture identique à la miniature de Marmion qui orne une des pages du Livre d'heures de Marie de Bourgogne ${ }^{20}$ (fig. 5, 6). Une étude attentive montre que si l'esprit "marmionesque» est bien là, la plupart des miniatures ne reflètent ni sa facture, ni sa palette reconnaissable. La faiblesse de certaines compositions, de nombreuses figures, et les maladresses dans le rendu des draperies trahissent le travail d'un assistant (fig. 4) ${ }^{21}$. Les paysages dénudés de la Fuite en Egypte ou de la Visitation (fig. 1, 11), où l'ocre clair domine, ponctué de rares taches vert pâle, sont impossibles chez Simon Marmion et rappellent plutôt les compositions du Second Maître des Grandes Chroniques de France $^{22}$. Cependant, certaines miniatures qui sont pourtant l'œuvre du même peintre sortent du lot par la qualité de leur exécution. Alors pourquoi font-elles penser à une intervention de Marmion?

Nous avons en fait, avec ce manuscrit, un ensemble exceptionnel de modèles d'atelier. Quand le miniaturiste s'en éloigne, il crée des compositions médiocres comme la

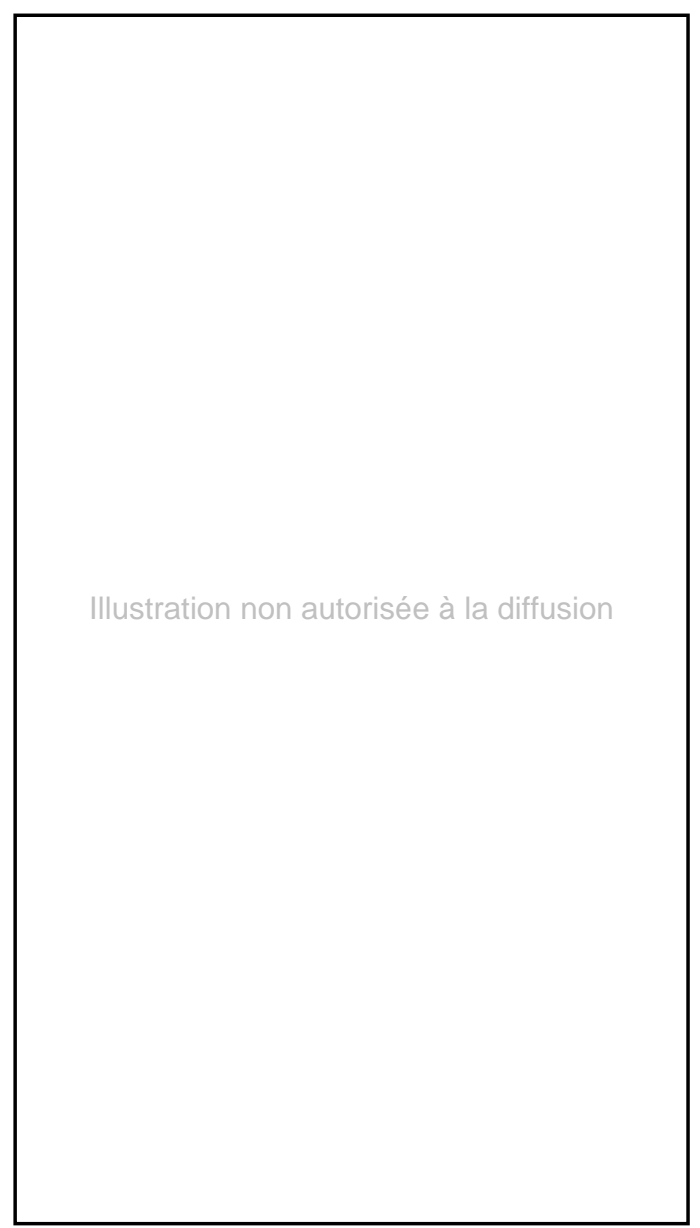

4. Atelier de Simon Marmion, Présentation ou temple, Paris, Bibliothèque nationale de France, n.a.lat. 3214, fol. $67 v^{\circ}$.

5. Atelier de Simon Marmion, Vierge à l'Enfant, Paris, Bibliothèque nationale de France, n.a.lat. 3214, fol. $86 v^{\circ}$

Présentation au temple (fig. 4). Les autres miniatures copient fidèlement les modèles du maître qu'il est facile de retrouver dans plusieurs œuvres attribuées à Marmion luimême : cinq livres d'heures, les Heures Berlaymont, Huth, Salting, Rolin et les Heures dites d'Henri VIII ${ }^{23}$, ainsi qu'une Crucifixion, miniature découpée conservée à Amsterdam ${ }^{24}$, sans oublier la Vierge à l'Enfant du manuscrit viennois. Toutes ces œuvres sont à peu près datables.

La première de ce groupe est le livre d'heures dit d'Henri VIII dont nous pouvons situer l'exécution de la décoration secondaire vers $1457^{25}$. Sans doute à la même époque que les miniatures de Tournai, fut commandé le livre d'heures destinéà Guillaume Rolin, fils du chancelier du duc de Bourgogne, Nicolas Rolin. Ce livre, dont certaines initiales contiennent les armoiries Rolin brisées d'un lambel à trois pendants d'argent ${ }^{26}$, peut être daté avant la mort du chancelier survenue en

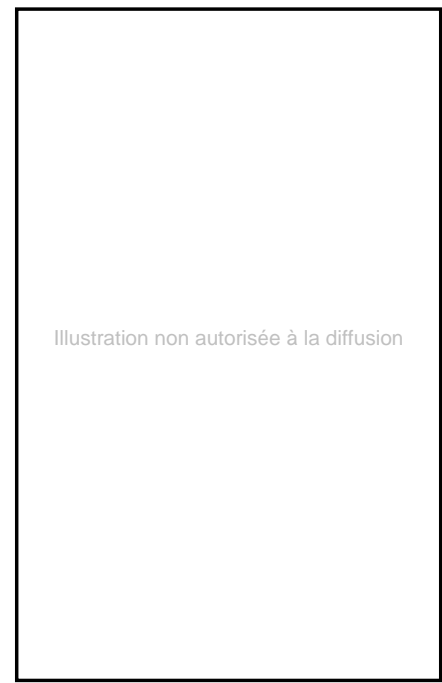

6. Simon Marmion, Vierge à l'Enfant, Vienne, Österreichische Nationalbibliothek, cod. 1857, fol. $35 \mathrm{v}^{\circ}$.

1462. Comme le premier manuscrit, il est illustré en grisaille complète, mais sans décoration marginale. On retrouve, dans ces deux livres d'heures, le même type

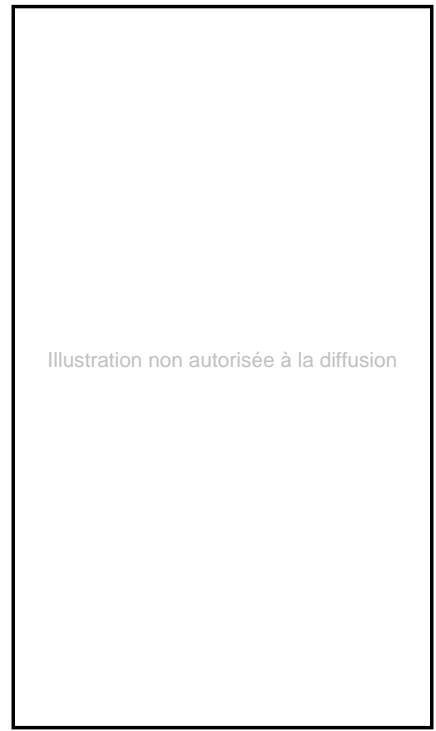

7. Atelier de Simon Marmion, Crucifixion, Paris, Bibliorhèque nationale de France. n.a. lat. 3214 , fol. $13 \mathrm{v}^{\circ}$.

de premier encadrement, constitué d'un double filet, à l'extérieur or et à l'intérieur bleu ou rouge piqueté d'or. Le côté supérieur cintré est orné d'une série de petits crochets 


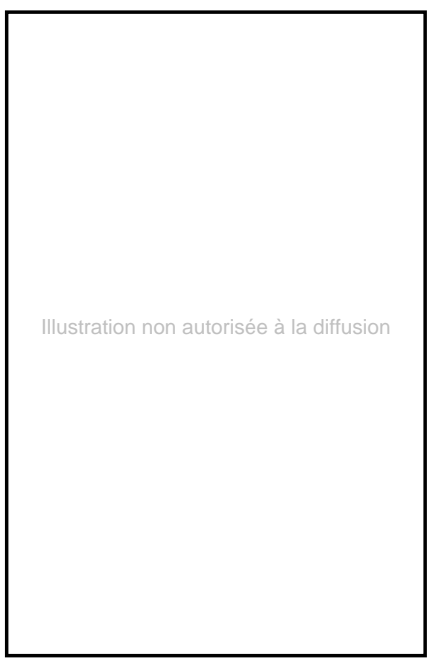

8. Simon Marmion, Crucifixion, San Marino, Huntington Library, ms. HM 1173, fol. 24 .

noirs, plus ou moins recourbés, et surmonté en son centre d'un minuscule rond. Ces petits crochets semblent être un motif décoratif propre à l'atelier Marmion ${ }^{27}$.

La Crucifixion d'Amsterdam paraît avoir été peinte dans les mêmes années que les deux manuscrits précédents $^{28}$. Les Heures Berlaymont ont été enluminées vers 1470$1475^{29}$, suivies par les Heures Salting et $H u t h$ que la critique place vers $1475-1480^{30}$. Enfin Otto Pächt a daté le livre d'heures destinéà Marie de Bourgogne, fille de Charles le Téméraire, vers $1475^{31}$.

Notre livre d'heures, pratiquement contemporain des Heures de Tournai, du feuillet d'Amsterdam et des Heures Rolin, se situe donc vers le début de cette production de livres d'heures. Si chacune de ses miniatures peut se prêter au jeu des comparaisons, nous ne prendrons que les plus frappantes. La première est bien sûr la Vierge à l'Enfant trônant entre deux anges musiciens, pareille en tout point à la miniature de Marmion du manuscrit viennois ${ }^{32}$ (fig. 5, 6). Un modèle devait sans aucun doute exister dans l'atelier depuis le début de la décennie soixante ${ }^{33}$. La scène de la Nativitéest identique à celle des Heures d'Henri VIII et des Heures Huth. L'Annonce aux bergers est une image inversée et simplifiée de la même peinture dans les Heures Huth, et la Fuite en Egypte (fig. 1) est à rapprocher de celle des Heures Berlaymont: Joseph et la Vierge sur

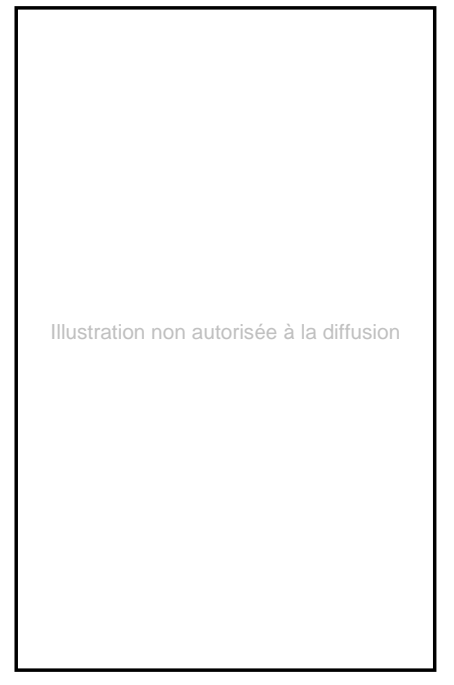

9. Simon Marmion, Crucifixion, Amsterdam, Rijksprentenkabinet, Rijksmuseum, inv. 1970, fol. 46.

l'âne ont les mêmes dimensions dans les deux miniatures. La Crucifixion dérive comme les autres Crucifixions de Marmion de celle du Pontifical de Sens ${ }^{34}$ ou d'un modèle commun qui pourrait bien être le Calvaire peint pour la salle de justice de l'hôtel de ville d'Amiens en $1454^{35}$. Elle se présente comme un condensé de motifs d'atelier que l'on retrouve en particulier dans les $\mathrm{Heu}$ res Berlaymont et dans le feuillet d'Amsterdam (fig. 7, 8, 9) : l'identité de modèle pour la Vierge et pour l'homme de dos au premier plan entre le feuillet d'Amsterdam et la miniature parisienne est particulièrement frappante ${ }^{36}$.

La très belle miniature de David en prière (fig. 10) se retrouve à la fois dans les Heures Salting, Berlaymont et les Heures d'Henri VIII ${ }^{37}$. Elle est reprise à la même époque par le Maître de Rambures dans trois livres d'heures $^{38}$.

Une autre image est commune au disciple de Marmion et au Maître de Rambures: la Visitation ${ }^{39}$ dans laquelle Elisabeth porte une coiffe de moniale, un chaperon à pointe, bordé de fourrure (fig. 11). A la même époque, ce détail qui semble d'origine picarde ${ }^{40}$ est utilisé par l'enlumineur, lui aussi formé en Picardie, qui a peint les miniatures du livre d'heures destiné à Isabeau de Roubaix $^{41}$ (fig. 12, 13). Une belle ferme, typique cense picarde, orne le fond de la miniature parisienne: nous avons là un autre modèle mar- mionesque dont témoigne le paysage de la Visitation des Heures Berlaymont (f' 35$)$.

Enfin il nous faut évoquer la miniature de la Pentecôte (fig. 14). La Vierge et les apôtres sont réunis dans une pièce largement ouverte sur une cour intérieure par deux baies et une arcade. Un escalier de quatre marches, soutenu par une demi-arche, permet d'accéder à la pièce et, au fond de la cour, un porche de brique donne sur la rue. Un apôtre agenouillé se tient dans cette cour et deux autres sous le porche. Marmion a peint une variante simplifiée de cette image dans un seul manuscrit connu, conservé à New York ${ }^{42}$. En revanche, cette image très particulière a servi de modèle à deux enlumineurs très importants du dernier quart du XVe siècle, le Maître du livre d'heures de Dresde et le Maître d'Antoine Rolin.

Installé à Bruges, alors que Marmion est à Valenciennes, le premier enlumineur a terminé la décoration des Heures Louthe, laissée inachevée par Marmion, et complété le livre d'heures Salting dont quelques miniatures avaient été commandées au peintre valenciennois ${ }^{43}$. Dans son livre d'heures éponyme, il reprend à la fois l'édicule de la Pentecôte du livre d'heures new yorkais et le porche ouvrant sur la rue avec les deux personnages de la miniature parisienne ${ }^{44}$

Quant au Maître d'Antoine Rolin, Anne-Marie Legarét5 a clairement montré ce qu'il devait à Simon Marmion. Cet enlumineur dont l'activité en Hainaut est connue à partir de $1490^{46}$ pourrait bien avoir fait son apprentissage à Valenciennes chez Marmion, dans le répertoire duquel il puise la plupart des sujets qui illustrent ses livres d'heures. Dans toute une série de Pentecôtes qui représentent chez lui un véritable lieu commun, il emprunte à la miniature de nos Heures parisiennes, l'arche supportant l'escalier et l'apôtre isolé dans la cour ${ }^{47}$. Ce qu'Anne-Marie Legaré pensait être l'un des rares motifs originaux de l'artiste n'est qu'un emprunt de plus à un modèle marmionesque.

Le Maître d'Antoine Rolin pourrait bien avoir hérité des cahiers de modèles de l'atelier de Marmion. Trois noms nous viennent à l'esprit, à son propos: Jan Prévost, Marion Marmion et Michel Clauwet. Le premier a épousé la veuve de Marmion, mais son style n'a rien à voir avec celui du Maître d'Antoine Rolin. Marie ou Marion Marmion, fille unique du peintre et «enlumineuresse", est morte jeune avant 1505 , année où s'ouvre la succession de son père ${ }^{48}$; comme le Maître d'Antoine Rolin semble poursuivre son activité après 1510 , il semble impossible de retenir cette seconde hypothèse. Michel Clauwet, quant à lui, est le fils de la sœur de Marmion, mariée en 1449 à Amiens. Clauwet est peintre et s'installe à Valenciennes où il est cité entre 1492 et $1519^{49}$. L'hypothèse Clauwet est séduisante mais, pour le moment, rien ne permet de l'étayer.

Ainsi avec ce manuscrit inédit, simple et pourtant belle production d'un collaborateur, c'est le fonctionnement même de cet atelier de Simon Marmion à Valenciennes qui prend forme devant nos yeux. Nous découvrons l'art original d'un peintre de bordures associé au maitre, vers 1465-1470, et certains motifs iconographiques ainsi que les petites figures des marges font apparaître un collaborateur de Marmion fortement ancré dans le milieu des enlumineurs de Picardie. Un disciple qui pourrait bien avoir émigré en même temps que le Maître. L'attribution de ce livre d'heures à l'atelier de Marmion vient conforter les conclusions de Sandra Hindman sur les deux feuillets de la collection Lehman; les miniatures, riche catalogue de modèles, confirment, s'il en était besoin, que d'une part de réels échanges artistiques existaient entre l'atelier de Marmion et les enlumineurs brugeois, tel le Maître du livre d'heures de Dresde, peut-être un picard émigré en Flandre ${ }^{50}$ et que, d'autre part, Marmion avait gardé un contact avec son milieu d'origine, en particulier avec un autre picard resté dans sa province, le Maître de Rambures. Enfin, les miniatures réaffirment le lien étroit, déjà mis en lumière par A.-M. Legaré, qui unit le Maître d'Antoine Rolin à l'atelier de Marmion, le premier apparaissant comme l'héritier des modèles du Maître et peut-être même de son atelier. 


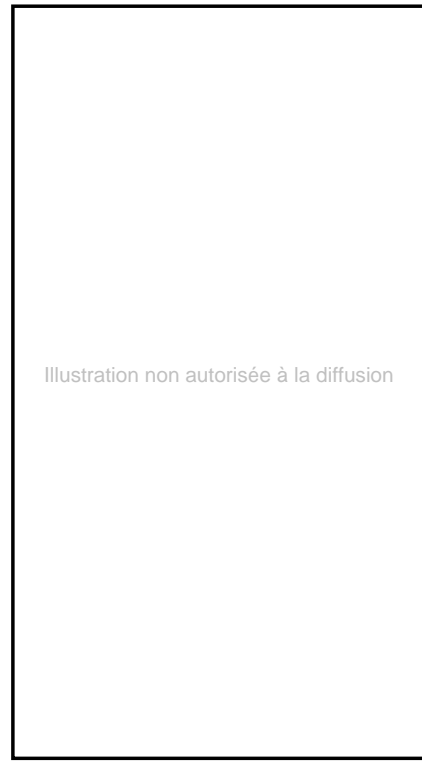

10. Atelier de Simon Marmion, David en prière, Paris, Bibliothèque nationale de France, n.a. lat. 3214 , fol. $116 \mathrm{v}^{\circ}$

\section{NOTES}

1. Thomas Kren éditeur, Margaret of York, Simon Marmion and "The Vision of Tondal", Malibu, 1990 (1992)

2. "Two Leaves from an Unknown Breviary, The Case for Simon Marmion ", Margaret of York p. 223-232. New York, Metropolitan Museum of Art, coll. Lehman, Ms. 80 (Les Vierges entrant au paradis); et localisation inconnue.

3. Pour une première approche de son fonctionnement, nous renvoyons à la communication de Bodo Brinkmann, lors du même colloque, "The Contribution of Simon Mar mion to Books of Hours from Ghent and Bruges", p. 181-194. B. Brinkmann a montré que Marmion ne se déplaçait pas, mais envoyait les feuillets peints à Bruges ou à Gand, où ceux-ci étaient adaptés au forma du livre, avant d'être éventuellement ornés de bordures.

4. N. acq. lat. 3214 : ff. $A+184+B, 182 \times$ $106 \mathrm{~mm}(95 \times 69 \mathrm{~mm})$.; ćcriture gothique de forme à 15 longues lignes; texte en latin; calendrier et certaincs rubriques en français douze miniatures, ff. 13v", Crucifixion, 18v", Pentecôte, 23v", Annonciation, 41v", Visita-

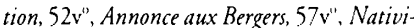
té, 62v", Adoration des mages, 67v", Présentation au Temple, $72 v^{\prime \prime}$, Fuite en Egypte, $80 \mathrm{v}^{\prime \prime}$, Couronnement de la Vierge, $86 \mathrm{v}^{\prime \prime}$, Vierge à l'Enfant assise sur un trône, entre deux anges musiciens, sur fond de paysage urbain, 116v", Daviden prière. A ces douze miniatures il faut ajouter celle, disparue, qui devait introduire les vigiles des morts. Il s'agissait peut-être d'une Résurrection de Lazare, sujet de tradition dans les provinces du Nord. Le relieur du XVII siècle a placé devant le premier folio de cet office (f' 117) la miniature de Daviden prière qui introduisait à l'origine les psaumes pénitentiaux (f'95).

5. Beaux livres anciens, cat de vente, Paris, Drount-Richelieu, 16 déc. 1994, n" 3. Nous tenons à remercier particulic̀rement François

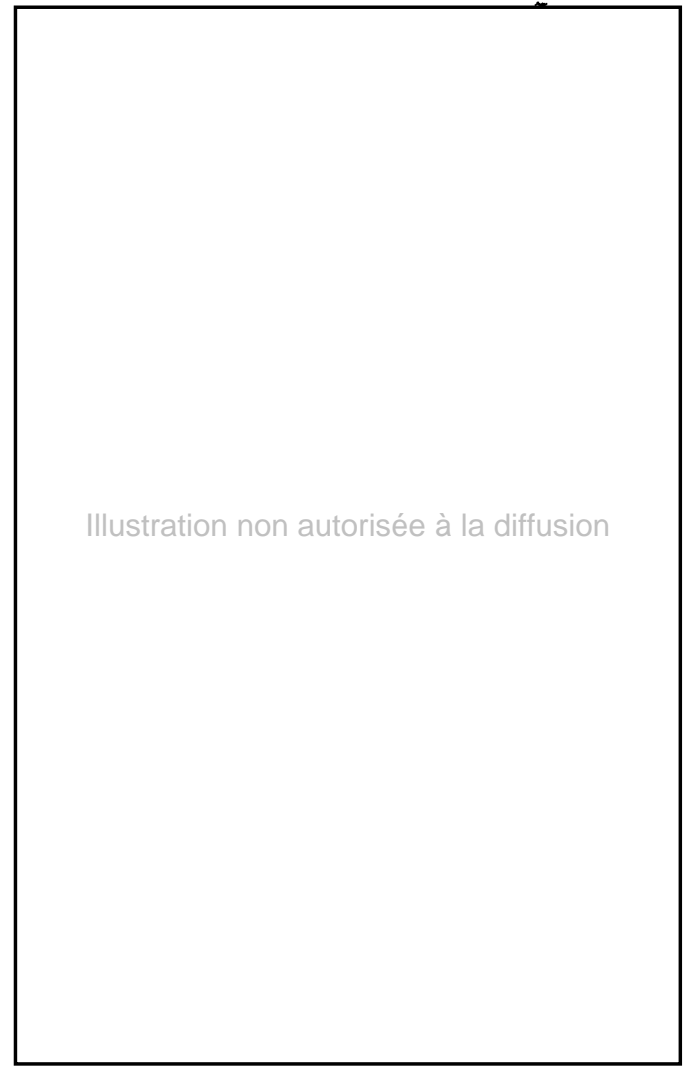

11. Atelier de Simon Marmion, Visitation, Paris, Bibliothèque nationale de France, n.a. lat. 3214, fol. $41 v^{\circ}$

Avril qui nous a permis l'étude de cette nouvelle acquisition de la Bibliothèque nationale de France.

6. f'A : Ce livre a esté donné par Nicolas Polcher a Anne Anthoinette Le Goeule sa belle fille. celuy et cela qui le trouveront estant égarez ou perdu luy rendront sans aucune façon et seron fort bien récompensé comme elle promet sous sa signature que voyez: Anne Anthoinette Le Goeule, demeurant a Feignies. 1706. Jésus Ma-

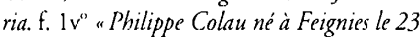
mai 1800 ". f. Bv": "Théodore Colau de Feignies". f. B : "Ce livre a été donné par mon oncle Théodore Colau à Colau ()ctave". Contre-plat inf. : "Ce livre a été donné par Colau Théodore à son neveu Octave Colau le 26 février 1854. T. Colau”.

7. Ordre inusité ailleurs, sauf dans certain livres d'heures à l'usage de 'l'royes: M. Gil, Le livre d'heures d'Isabeau de Roubaix ms. 6 de la bibliothéque municipale de Roubaix, mémoire de maitrise, inédit, université de Lille III, 1992, p. 64-65. Voir également L.M.J. Delaissć, "The Importance of Books of Hours for the History of Medieval Books ", Gathering for Dorothy E. Miner, Baltimore, 1974 , p. 207

8. En Flandre, les thèmes pour ces deux séquences sont généralement lc Massacre des Innocents et le Jugement dernier; D. Vanwijnsberghe, "The Cyclical Illustrations of The Little Hours of Virgin in Pre-Evckian Manuscripes ", Flanders in European Perspective, Manuscript Illumination around 1400 in Flanders and Abroad (1993), Louvain, 1995. p. $286-287$.

9. Heures dites d'Henri VIII, Tournai, Bibliotheque municipale, ms. 122; Heures Berlaymont. San Marino, Huntington Libra ry, ms. HM. 1173; Turin, Musco Civico, ms. 558.

10. Amiens, Bibliothèque municipale, ms. 200 .

11. En rouge, "Bertremieu", Martin, "Andrieu ", "Nicholay", Nicaise et Ftienne. Fn noir, Audegonde, sa sour Gertrude, Adrien. Quentin au 2 mai, mais pas au 31 octobre Eloy, Gilles, Bertin, Lambert; Remy, Gillain, Crispin, Hubert, Liénart

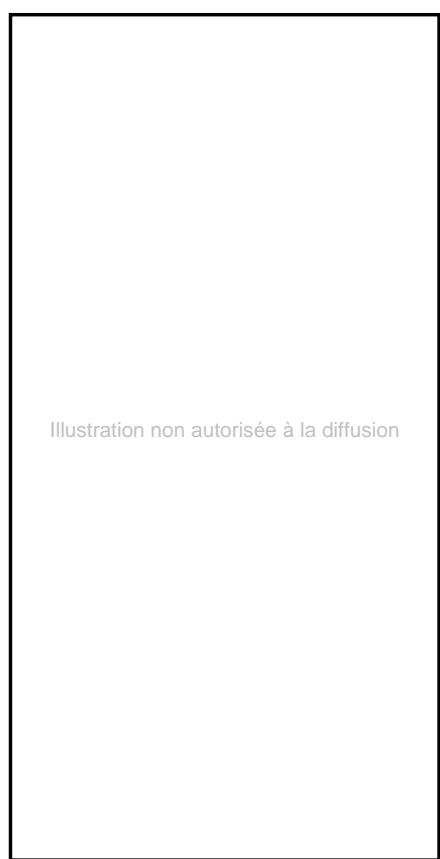

12. Enlumineur anonyme picard Visitation, Paris, Bibliotheque nationale de France, lat. 13263, fol. 52
12. Pour Amiens, plus particulièrement, Fremin ( 2 fois), Fremin er Léri, Waleri, Honoré, Fuscien ( 2 fois). Pour Thérouanne Arras, Cambrai et Tournai, Adrien, Liffart (I.iéphard), Amand, Cornille, Omer, Légier et Quentin, et deux incursions dans le diocèse de Tournai pour saint Bavon de Gand au $1^{\mathrm{t}}$ novembre et saint Donas, peut-être de Bruges, au 14 du même mois au lieu du 6 août.

13. Litanies en latin, ff. $105 v^{\prime \prime}-114 v^{\prime \prime}$. On peur noter, Corneille, Denis, Romuald (Romualdus, Rombaud de Malines), Lambert, Winoc, Fursy, Amand, Vaast, Martin (2 fois), Adrien, Nicolas, Gilles, Eloy, Rémi et Bavon, pour les saints et Ursule, Brigide, Geneviève, Apolline, Hélène, Aldegonde et Gertrude pour les saintes. Les suffrages sont peu significatifs: la présence des saints Quentin, Adrien et Fiacre ne nous dirige pas vers un diocèse septentrional précis.

14. L'absence de sainte Waudru semble exclure la ville de Mons.

15. Le rapprochement que fait Sandra Hindman entre la décoration secondaire du feuillet de New York et celle des Heures Salting nous paraît peu probant (art. cit. à la note 2 , p. 227). B. Brinkmann, art. cit. à la note 3 , p. 184, a par ailleurs montré que les Heures Salting appartiennent à la production typiquement brugeoise et leur décor marginal est l'ceuvre de deux enlumineurs brugeois, le Maître du livre d'heures de Dresde et le Maître du manuscrit Fitzwilliam 268. Marmion fut seulement chargé de peindre certaines miniatures.

16. Saint-Pétersbourg, Bibliothèque nationale de Russie, Erm. 88, f'l, reproduit page 81 de la seconde édition du catalogue d'exposition, Fr. Avril, N. Reynaud, L.es Manuscrits a peintures en France, 1440-1520, Paris, 1994.

17. Roucn, Bibliothèque municipale, ms. I. 2

18. Marmion est payé par l'intermédiaire de l'évêque de Salisbury qui se charge de faire

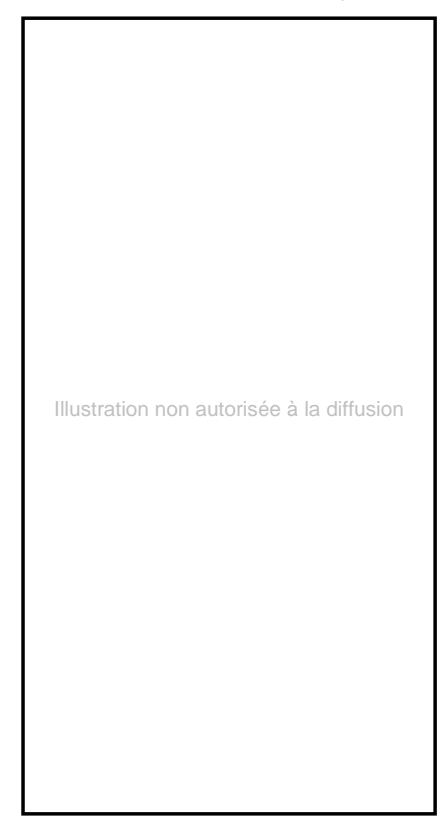

13. Maitre d'Isabeau de Roubaix Visitation, Roubaix, Bibliothèque municipale, ms. 6, fol. $54 \mathrm{v}^{\circ}$. 
relier le livre, voir pour le détail du paiement, M. Hénaule "Les Jchan, Simon, Mille et Colinet Marmion, peintre amiénois du $\mathrm{XV}$ " siècle ", Revue archéologique, 4" série, IX, 1907, p. 419. Le fait que l'évêque de Salisbury a servi d'intermédiaire dans la transaction et que l'office des morts du livre d'heures parisien est peut-être à l'usage de Sarum est sans doute pure coïncidence.

19. Il suffit pour s'en convaincre de rapprocher certains encadrements de notre livre d'heures parisien des très beaux feuillets enluminés de deux livres d'heures exécutés en Picardie (Amiens ou Hesdin?) par le Maitre de Rambures, alors que Marmion est déjà à Valenciennes: Amiens, Bm., ms. 200, vers 1460-1465; New York, Pierpont Morgan Library, ms. 194, vers 1470-1475.

20. Vienne, Österreichische Nationalbibliothek, cod. 1857, f' 35v".

21. On peut toutefois se demander si certains visages, comme celui de la Vierge de l'Adoration des mages, ou des figures enticres, comme David et Nathan au folio $116 \mathrm{v}^{\circ}$, n'ont pas été réservés au maitre lui-même.

22. Pour plus de détails sur cet enlumineur, voir $\mathrm{N}$. Reynaud, op. cit. à la note 16, p. 84 et 90-91.

23. San Marino, Huntington Library, ms. HM. 1173; Londres, British Library, ms. Add. 38126 er Victoria and Albert Museum, Ms. Salting 1221 ; Madrid, Biblioteca nacional, Ms. Res. 149; Tournai, Bibl. mun., ms. 122.

24. Amsterdam, Rijksprentenkabinet (Rijksmuscum), Inv. $1970: 46$.

25. En effet, l'enluminure des bordures et du texte est l'œuuvre d'un arclier à qui l'on doir la décoration des marges et des grandes initiales du Traité de l'Oraison dominicale, traduit et copié pour Philippe le Bon par Jean Miélot à Lille entre 1456 et 1457 , Bruxelles, Bibliothèque royale, ms. 9092. La décoration secondaire des Heures de Tournai, assez voyante, jure avec la grande finesse et l'élégance des miniatures en grisaille, ce qui pourrait laisser penser que le manuscrit, production du commerce laissée inachevée après l'exécution des bordures (f" 36 , écu vierge), ne fut confić qu'un peu plus tard à Simon Marmion. Cependant, les miniatures sont encore très proches des productions de l'artiste de la décennie 50 : vastes paysages accidentés où l'eau est partout présente, présence du haut rocher en pain de sucre placé à droite de la composition, canon allongé des figures au caractère animé (très rare chez les personnages de Marmion), mais surtout l'architecture intéricure de l'Annonciation ( $\mathrm{f}^{\mathrm{f}}$ 36) s'ouvrant à droite sur un cloître identique à celui peint sur le volet droit du Retable de Saint-Bertin, conservé à Berlin.

26. Indication de Nicole Reynaud que nous remercions chaleureusement pour nous avoir éclairé de ses conseils judicieux tout au long de cette ćtude.

27. Voir la Crucifixion du Livre de prières de Philippe le Bon (Paris, BnF., n. a. fr. 16428) un feuillet arraché à un livre d'heures (vers 1470, Paris, Ecole nationale des beaux-arts, M. 130) er la Visitation de nos Heures pari-

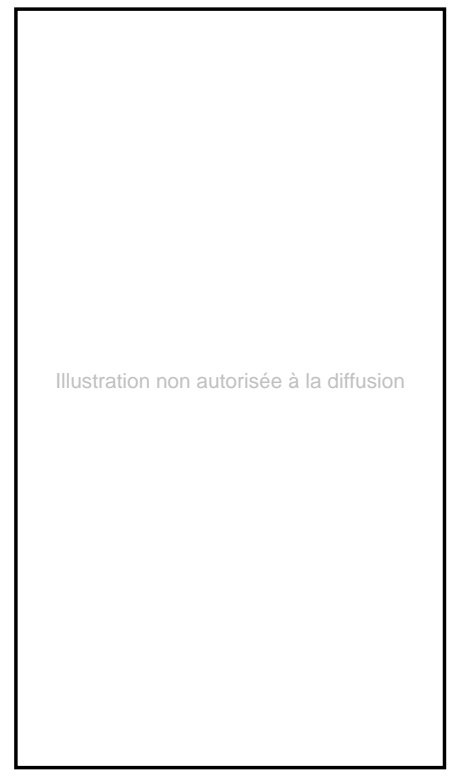

14. Atelier de Simon Marmion, Pentecôte, Paris, Bibliotheque nationale de France, n.a. lat. 3214 , fol. $18 \mathrm{v}^{\circ}$.

siennes. Mais seuls les miniatures de Tournai et Madrid ont en plus le piqueté d'or sur le filet coloré.

28. N. Reynaud, op. cit. à la note 16, p. 88 , la rapproche de la Crucifixion du Livre de prières de Philippe le Bon, manuscrit exécuté, d'après le texte, peu après 1462. Th. Kren, dans un article récent, a rapproché cette miniature et trois autres venant du même livre, d'une suite de huit miniatures conservées à la British Library, reconstituant ainsi les membres éparts d'un livre d'heures exécuté par Marmion et son atelier, et a daté l'ensemble de la décennie 60: "Some Newly Discovered Miniatures by Simon Marmion and his Workshop ", The British Library Journal, 22, n" 2, 1996, p. 193-219.

29. Idem, p. 89.

30. Pour les Heures Huth, Th. Kren, Renaissance Painting in Manuscripts, Treasures from The British Library, New York, 1983, n 4 p. 31-39, ce pour les Heures Salting, G. Clark "The Chronology of Lourhe Master and his Identification with Simon Marmion ", Mar garet of York cité à la note 1, p. 200: la chronologie établic par l'auteur pour les livres d'heures doit être en partie révisée.

31. O. Pächt, D. Thoss, U. Jenni, Die Illuminierten Handschriften und Inkunabeln der Österreichischen Nationalbibliothek. Flamische Schule, II, 2 vol., Vienne, 1990, I, p. 69-85, fig. 55-75, II, pl. X et 102-150.

32. Iconographie, dimensions, couleurs. Les deux images sont superposables sauf pour la place des anges qui sont légèrement plus proches du trône dans la miniature parisienne, plus étroite que celle de Vienne (56 e $67 \mathrm{~mm}$ ).

33. On peut s'interroger sur la date d'exécution de la miniature de Vienne et donc sur celle du manuscrit lui-même.

34. Bruxelles, Bibliothèque royale, ms. $9215, f^{\prime \prime} 129$.
35. "A Simonnet Marmion, paintre(...) pour sa peine et salaire d'avoir paint et ouvré ung tableau où est figuré la représentation de Nostre Seingeur, Nostre Dame, sainct Jehan et autres personnages(...) ", Hénault, art. cit. à la note $18, n^{\prime \prime} 10$, p. 412 .

36. La Vierge parisienne est assez proche de la Vierge éplorée de la Crucifixion peinte sur bois par un disciple de Marmion, Philadelphie, Museum of Art, collection John G Johnson : Margaret of York, op. cit. à la note 1, fig. 237, p. 246.

37. Le détail du bateau à voile n'existe que dans notre livre parisien et celui de Londres et la figure de Natan ne se trouve dans aucun livre d'heures connu de Marmion : initiative du collaborateur, ou bien le modèle marmionesque a-t-il disparu? Le même sujet assez rare de David en prière sermonné par Natan introduit les psaumes pénitentiaux d'un livre d'heures à l'usage d'Amiens (vers 1470), conservé à la bibliothèque municipale d'Arras, ms. 515, f' 137. Nous avons ici une contamination de l'image traditionnelle et assez banale de David en prière (dans un paysage ou sur une terrasse) par celle de David assis sur son trône et sermonné par Natan, que l'on rencontre le plus souvent dans les manuscrits flamands.

38. Souligné par G. Clark, art. cit. à la note 1, p. 202, fig. 175; pour les deux premiers manuscrits, voir à la note 19; le dernier exécuté peu après 1473 , est à Londres, British Library, ms. Add. 19738.

39. Londres, British Library, ms. Add 19738, f 42; Marmion, lui-même, reprend cette iconographie dans les Heures La Flora de Naples (Bibl. nazionale, ms. 1 B 51, vers 1490), la Vierge et sainte Elisabeth sont cadrées en buste.

40. Un livre d'heures à l'usage d'Amiens du milieu du $\mathrm{XV}^{c}$ siècle: Paris, BnF., ms. lat. $13263, f^{\circ} 42$.

41. Roubaix, bibliothèque municipale, ms. $6, f^{\prime} 54 v^{\circ}$; ce manuscrit a été exécuté vers 1464-1465, au moment du mariage d'Isabeau de Roubaix ( $\uparrow 1502$ ), héritière de la riche seigneurie de Roubaix, avec le frère cadet du comte de Saint-Pol, Jacques de Luxembourg († 1487). L'iconographie générale (sans le détail du chaperon) dérive bien sûr de Van der Weyden, voir la Visitation de Leipzig.

42. Pierpont Morgan Library, ms. $6, f^{\prime} 72 v^{\circ}$ (Clark, art. cit. à la note 30, p. 204, fig. 179). Dans les autres livres d'heures (sept cités par Clark) la scène se passe de manière traditionnelle dans une pièce assez exiguë.

43. Louvain-la-Neuve, Université catholique, ms. A. 2 : Brinkmann, art. cit. à la note 3, p. 182 et 184 .

44. Dresde, Sächsische Landesbibliothek, Ms. A. $311, f^{\prime} 19 v^{\circ}$, reproduit dans Margaret of York, op. cit. à la note 1, p. 204, fig. 180.

45. Margaret of York, p. 209-222, et en dernier lieu, "L'héritage de Simon Marmion en Hainaut (1490-1520) „, Valenciennes aux XIV et XV siècles. Art et Histoire, Valenciennes, 1996, p. 201-224.

46. La plupart des clients du Maître d'Antoine Rolin et de ses collaborateurs sont ori- ginaires de Mons ou de sa région, Legaré, 1996, p. 204-216.

47. La Haye, Koninkliike Bibliothek, ms. 76 $\mathrm{f} 16, \mathrm{f}^{\prime} 31$, reproduit dans Margaret of York, op. cit. à la note 1 , fig. 193.

48. Hénault, art. cit. à la note 18, p. 129 131.

49. Reynaud, op. cit. à la note 16, p. 80 et Hénault, art. cit., pièces justificatives $n^{\circ} 81$ 109.

50. Les miniatures de Marmion semblent avoir eu quelque succès à Bruges : vers 1470 un enlumineur anonyme (Lievin van Lathem ?) reproduit la crucifixion du Pontifical de Sens dans un livre d'heures à l'usage de Rome, avec calendrier pour Mons : Oxford, Bodleian Library, ms. Gouth Liturg. 15, f'14.

Marc GIL, chargé de cours d'histoire de l'art médiéval, université Lille III - Charles-de-Gaulle 59653 Villeneuve d'Asq Cedex. 